\title{
51 Endurance training and the large blood vessels
}

Regular exercise increases the blood volume in the veins and arteries, which in turn improves the viscosity of the blood because it lowers the concentrations of clotting factors like fibrinogens while increasing the amount of tissue-specific plasminogen activators. The transportation of oxygen and temperature regulation are improved.

The interior surface of the blood vessels, the endothelium, also plays a key role in improving blood vessel function during regular endurance exercise. This cell layer then produces more nitrogen monoxide and prostacyclin ( $\downarrow$ Chapter 15 ). Both substances cause the blood vessels to dilate and, as a result, lower the blood pressure. Furthermore, prostacyclin inhibits platelet activation, thereby also reducing the risk of thrombosis. Finally, fewer inflammatory cells migrate into the blood vessel wall, which in turn is linked to a reduction in the arteriosclerosis risk.

The increased blood flow during exercise is thought to cause an increase in endothelial activity (Flammer et al. 2012). Its intensity can be easily measured by ultrasound as flow-mediated vasodilation, or FMD for short, and is a parameter for assessing endothelial function. The higher the elasticity of the arteries, the better the condition of the endothelium and the lower the risk of coronary heart disease and hypertension becomes ( $\downarrow$ Chapter 53).

In general, too much abdominal fat can reduce the FMD of the arteries (Romero-Corral et al. 2010).

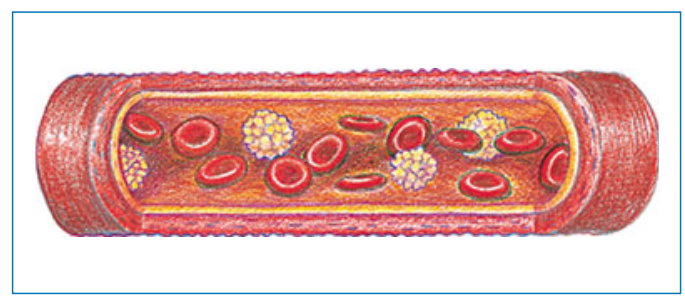

Fig. $\mathbf{5 1 . 1}$

Endurance athletes therefore also benefit from their strenuous exercise in terms of optimal endothelial function because they usually do not suffer from excessive accumulations of body fat. With minor limitations, these dual benefits of endurance training similarly apply to overweight people: When they engage in physical exercise their visceral fat deposits tend to be burned off first ( $\downarrow$ Chapter 56 ). The Women's Health Study conducted on 10,339 women over a mean observation period of 14 years showed that regular physical activity could considerably reduce the risk for hypertension in overweight women. However, these findings could not be transferred completely to the risk profile of normalweight women (Jackson et al. 2014).

The large blood vessels also benefit from plenty of exercise. 